\title{
Estudio Comparativo: Prevalencia Patologías Bucales en Pacientes Pediátricos Oncológicos 1997 - 2007
}

\author{
Comparative Study: Oral Pathologies Prevalence \\ in Pediatric Oncology Patients 1997 - 2007
}

Rodriguez, M.*; Manriquez, X.; Rojas, I. G."; Fernandez, E. ${ }^{* * *}$; Bretahuer, U.* \& Sepúlveda, E.*

RODRIGUEZ, M.; MANRIQUEZ, X.; ROJAS, I. G.; FERNANDEZ, E.; BRETAHUER, U. \& SEPÚLVEDA, E. Estudio comparativo: Prevalencia patologías bucales en pacientes pediátricos oncológicos 1997-2007. Int. J. Odontostomat., 4(2):149156, 2010.

RESUMEN: Los pacientes pediátricos oncológicos con frecuencia presentan lesiones orales debido a su neoplasia o como efecto colateral del tratamiento. El objetivo de este estudio fue comparar la prevalencia de patologías de la mucosa oral en niños con cáncer que fueron hospitalizados y tratados con quimioterapia en el Hospital Regional de Concepción, en los años 1997 y 2007. Se realizó un estudio descriptivo retrospectivo longitudinal en datas de 148 pacientes (74 cada año) con patologías neoplásicas en tratamiento con quimioterapia (Leucemias, linfomas, tumores del Sistema Nervioso Central y otros), registrando sus datos generales y la patología bucal (mucositis (M), candidiasis (C), lesiones por Virus Herpes tipo 1 (VHS) y síndromes hemorragíparos $(\mathrm{H})$. Los datos se resumieron en tablas anuales y fueron sometidos a análisis estadísticos. Se encontró una disminución significativa del número de pacientes con patologías bucales en el año 2007 en relación al año 1997 ( $P<0.05$, Tet de Fisher). Además se encontró una tendencia a la baja en los pacientes con candidiasis y con mucositis en el año 2007 en comparación con 1997. Es necesario seguir estudiando medidas para prevenir, diagnosticar y/ o tratar tempranamente las patologías orales de los pacientes en tratamiento antineoplásico.

PALABRAS CLAVE: leucemia, lesiones orales, quimioterapia.

\section{INTRODUCCIÓN}

El cáncer, es la segunda causa de muerte en el grupo etario de 5-9 años en Chile (Departamento de Epidemiología, 2005) y su tasa de mortalidad es de 3.4 por 100.000 niños menores de 15 años (Departamento de Epidemiología; Cerda et al., 2008). En Chile se espera alrededor de 517 casos nuevos cada año con una sobrevida del $73 \%$ después de 5 años de diagnosticada la enfermedad (Departamento de Epidemiología).

Los tipos de cáncer más comunes en niños son leucemias, linfomas y tumores del Sistema Nervioso Central (Cho et al., 2000). Las leucemias, linfoblástica aguda (LLA) y mieloblástica aguda (LMA) representan el $40 \%$ de los cánceres infantiles en Chile (Ministerio de Salud, 2005).
La LLA afecta a linfocitos. Sus células se acumulan en la médula ósea, reemplazan a las células sanguíneas sanas, y se diseminan a otros órganos. (Ministerio de Salud, 2005).

En la LMA se encuentra afectada una gran variedad de glóbulos blancos, granulocitos, monocitos y plaquetas que al igual que en la LLA, se acumulan en la médula ósea, reemplazando a las células normales para después diseminarse al resto del organismo (Ministerio de salud, 2005).

Los linfomas son un grupo de tumores malignos, diferentes tanto genéticamente como en su presentación clínica y tratamiento, que afectan al tejido linfoide (Cheson, 2004). En general se clasifican en linfomas Hodgkin y no Hodgkin (Cheson; Ultmann,

\footnotetext{
"Departamento de Patología y Diagnóstico, Facultad de Odontología, Universidad de Concepción,

* Departamento de Estomatología Quirúrgica, Facultad de Odontología, Universidad de Concepción

*** Servicio de Pediatría, Hospital Guillermo Grant Benavente

Financiado por proyecto DIUC 203.103.012-1.
} 
1971). En Chile, corresponden al $13 \%$ de los cánceres infantiles, se observan 50 casos nuevos por año y de preferencia en niños mayores de 4 años (Ministerio de Salud, 2004).

Los tumores del sistema nervioso central, corresponden al $17 \%$ de los tumores malignos infantiles (Ministerio de Salud, 2004).

Los principales tratamientos para el cáncer son la quimioterapia, las radiaciones ionizantes, la intervención quirúrgica y la inmunoterapia. Su indicación va a depender de la edad del paciente, origen, extensión del tumor, tipo histológico y grado de inmadurez (Diamond, 1959; Del Pozo \& Beresi, 1976).

Actualmente existen más de 100 drogas antineoplásicas, mejorando las expectativas y calidad de vida de los pacientes que padecen cáncer (Connor \& McDiarmid, 2006).

Las drogas utilizadas en la quimioterapia se clasifican según su mecanismo de acción en: antimetabolitos (metotrexato, 6-mercaptopurina, citarabina, 5-fluoruracilo); alquilantes (ciclofosfamida, BCNU-CCNU); antibióticos (actinomicina C, adriamicina); enzimas (L-asparraginasa) y corticoesteroides (prednisona) (Del Pozo \& Beresi).

Estas drogas son tóxicas sobre las células neoplásicas, pero además producen efectos colaterales, especialmente en niños, dependiendo de la droga, el tiempo y cantidad utilizada (Oeffinger \& Hudson, 2004). Los pacientes oncológicos en tratamiento con quimioterapia, frecuentemente presentan patologías orales las que pueden deberse a su enfermedad de base, a la potencialidad inmunosupresora de las terapias utilizadas y /o al efecto citotóxico directo que ellas tienen sobre la mucosa bucal. Las principales manifestaciones orales reportadas en un grupo de estudio semejante fueron: mucositis, candidiasis, lesiones herpéticas y síndromes hemorragíparos (Sepúlveda et al., 2000).

La mucositis oral, complicación común del tratamiento quimioterapéutico, disminuye la calidad de vida del paciente e incrementa el costo de cuidados de éste (Epstein et al., 2002; Lalla et al., 2008). Es una reacción inflamatoria que afecta a todo el tracto gastrointestinal y se clasifica fundamentalmente en dos tipos principales: eritematosa y ulcerativa (Lalla et al.). La mucositis ulcerativa es más grave y aparece a los siete días del inicio del tratamiento antineoplásico
(Castell et al., 2001). Muchas veces esta patología limita la terapia oncológica especialmente en tumores sólidos ( $51 \%$ desarrollaron mucositis oral), llegando a ser la complicación mas debilitante en el caso del trasplante (Lalla et al.).

Otra complicación frecuente en la mucosa oral, es el desarrollo de infecciones, principalmente por el virus del herpes simple tipo 1 (VHS1) o por Candida albicans, siendo mayor el riesgo en enfermos con neutropenia prolongada lo que puede comprometer la vida del paciente (Albert et al., 2006) reportaron que el diagnóstico clínico de las infecciones orales en los pacientes inmunocomprometidos puede ser difícil debido a su escasa respuesta inflamatoria y que las infecciones por hongos son 5 veces mayor que las bacterianas. La quimioterapia induce a la neutropenia, por lo que la colonización por hongos es considerada como el principal factor de riesgo de infección secundaria (Gozdasoglu et al., 1999).

La candidiasis es una complicación infecciosa aguda común en niños en períodos de neutropenia (Sonis \& Fey, 2002) por lo que se considera de vital importancia la prevención y el control de la infección local para prevenir candidiasis sistémicas (Epstein et al., 2003).

El VHS1, es el patógeno viral más común encontrado en lesiones bucales en pacientes que reciben quimioterapia. Reconocerlo es fundamental para tratarlo con el antiviral apropiado (Saral, 1990; Subramaniam et al., 2008). Las lesiones orales pueden ser expresión de una infección primaria o una reactivación del virus, la que puede ser sintomática (reagudización) o asintomática (recurrente) (Miller \& Danaher, 2008). El contagio es a través de contacto por secreciones ya infectadas en los primeros años de vida (Herget et al., 2005). Los linfocitos y sus citoquinas juegan un rol importante controlando la reactivación del VHS1 (Liu et al., 2000; Freeman et al., 2007), por lo tanto, cuando los linfocitos se encuentran comprometidos, es mayor la frecuencia de recurrencia del virus VHS1 (Sepúlveda et al., 2008).

Los síndromes hemorragíparos que incluyen petequias, equimosis, hematomas, y hemorragias pueden deberse al efecto trombopénico de las drogas sobre la médula ósea, ó a defectos cualitativos de las plaquetas u otros componentes del sistema de coagulación (Sepúlveda et al., 2000).

Todas estas patologías orales disminuyen la 
calidad de vida, aumentan el costo del tratamiento, prolongan hospitalizaciones, postergan quimioterapias y pueden dar lugar a enfermedades oportunistas. Esto hace comprometer al odontólogo en su estudio.

El objetivo de este estudio fue comparar la prevalencia de patologías de la mucosa oral en niños con cáncer, hospitalizados y tratados con quimioterapia en el Hospital Regional de Concepción, durante los años 1997 y 2007.

\section{MATERIAL Y MÉTODO}

Se realizó un estudio descriptivo, retrospectivo de la información registrada en fichas clínicas de 148 pacientes (74 cada año) para extraer datos generales del paciente (edad, sexo, enfermedad sistémica, número de consulta) y de la patología bucal asociada a su neoplasia o tratamiento (candidiasis $(C)$, mucositis post quimioterapia $(M)$, síndromes hemorragíparos $(H)$, lesiones por Virus Herpes Simple (VHS)).

Con respecto a las patologías bucales se determinó el número de pacientes que padecieron la enfermedad y el número de episodios registrados cada año.

Los datos se resumieron en tablas anuales y fueron sometidos a prueba estadística (Test de Fisher $\mathrm{P}<0.05)$.

\section{RESULTADOS}

Se registraron los datos de 74 pacientes cada año. En 1997: 28 mujeres y 46 hombres (promedio de edad= 6,6 \pm años). En el año 2007: 29 mujeres y 45 hombres (promedio de edad de 6.8 \pm años). Las patologías neoplásicas más frecuentes en ambos años fueron las LLA y SNC (Fig. 1). Las drogas utilizadas en los pacientes sometidos a quimioterapia en este estudio se detallan en la Tabla I.

Al analizar las diferencias entre la prevalencia de lesiones orales en 1997 vs. 2007 se encontró una disminución significativa en la prevalencia de enfermedades orales en el año 2007 al compararlo con 1997 (24/74 vs. 35/74). Por otra parte, al analizar en forma individual los tipos de patologías orales no se encontraron diferencias significativas entre ambos años, sin embargo se observó una tendencia a la baja en la prevalencia de candidiasis y mucositis (Tabla II).

\section{DISCUSION}

Este estudio tuvo como objetivo comparar las manifestaciones orales presentes en dos grupos semejantes con un intervalo de diez años de diferencia.

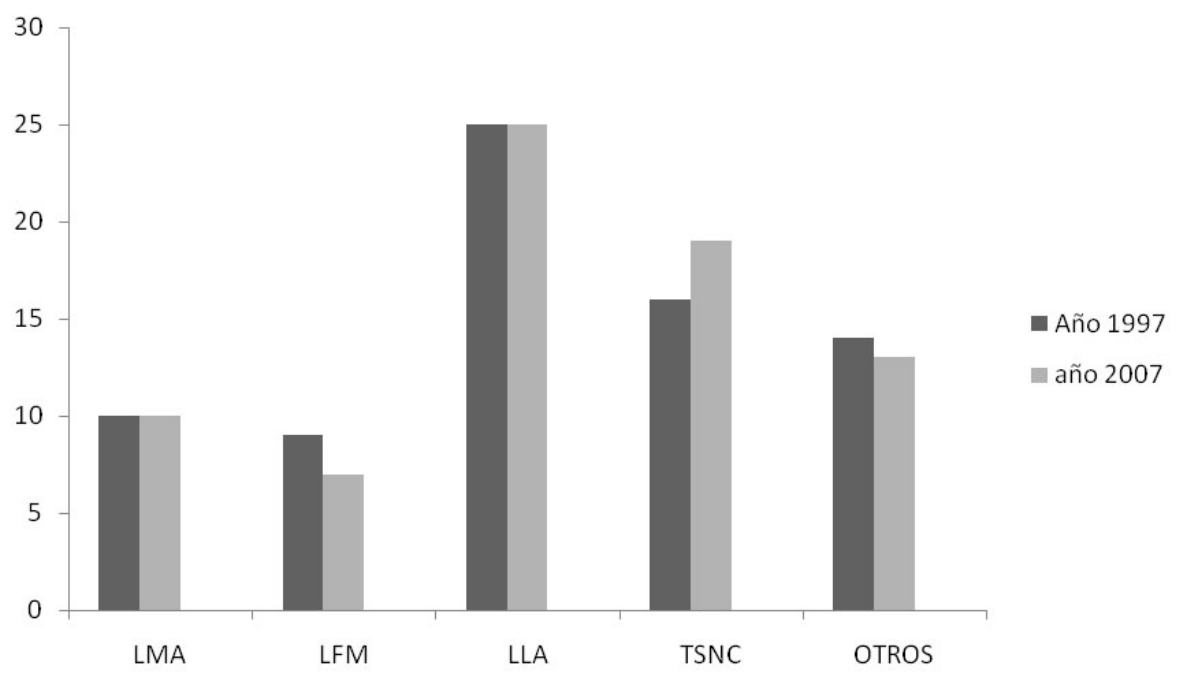

Fig. 1. Pacientes por patología neoplásica; años: 1997 y 2007. LLA, leucemia linfoblástica aguda; LMA, leucemia mieloblástica aguda; LFM, linfoma; RB, rabdomiosarcoma; TSNC, tumor del Sistema Nervioso Central. 


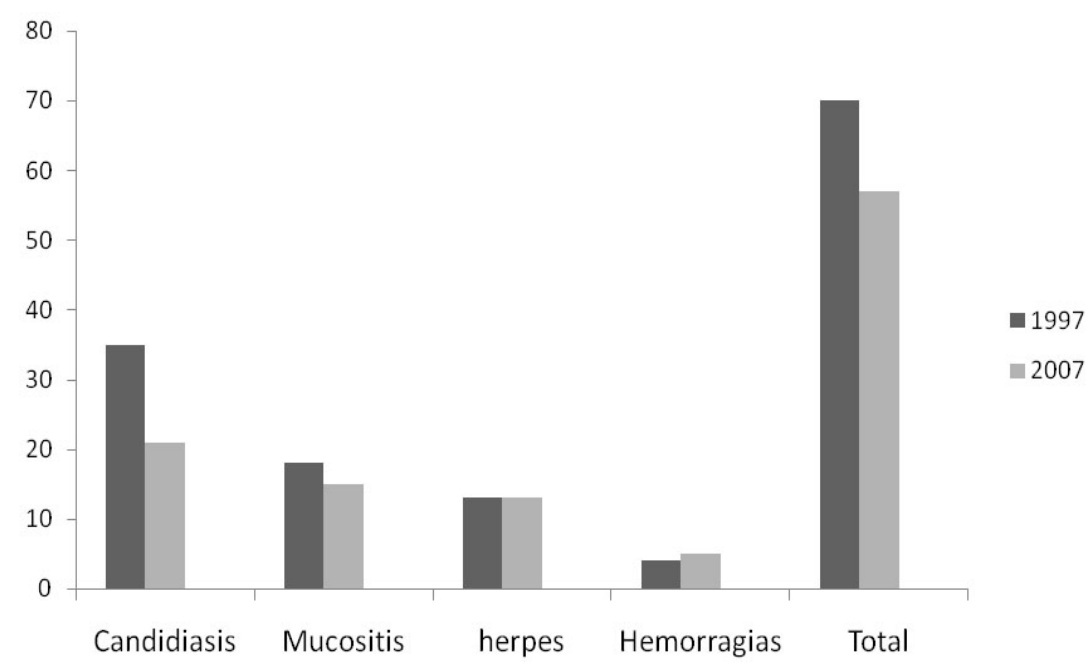

Fig. 2. Episodios por patología bucal; años1997 y 2007.

\begin{tabular}{lccccc}
\hline Drogas & LLA & LMA & LFM & RB & TSNC \\
Vincistrina & $\mathrm{X}$ & & $\mathrm{X}$ & $\mathrm{X}$ & $\mathrm{X}$ \\
Actinomicina & & & $\mathrm{X}$ & $\mathrm{X}$ & $\mathrm{X}$ \\
Ciclofosfamida & $\mathrm{X}$ & $\mathrm{X}$ & $\mathrm{X}$ & \\
Etopósido & & & $\mathrm{X}$ & $\mathrm{X}$ \\
Isofosfamida & & & $\mathrm{X}$ & \\
Cisplatino & & $\mathrm{X}$ & & \\
Nitrosurea & & $\mathrm{X}$ & \\
Carboplatino & & $\mathrm{X}$ & \\
Doxorrubicina & $\mathrm{X}$ & $\mathrm{X}$ & $\mathrm{X}$ & \\
Prednisona & $\mathrm{X}$ & $\mathrm{X}$ & $\mathrm{X}$ & \\
Daunorrubicina & $\mathrm{X}$ & $\mathrm{X}$ & $\mathrm{X}$ & \\
Aspariginasa & $\mathrm{X}$ & $\mathrm{X}$ & $\mathrm{X}$ & \\
Citarabina & $\mathrm{X}$ & $\mathrm{X}$ & $\mathrm{X}$ & \\
Purinetol & $\mathrm{X}$ & & & \\
Metotrexato & $\mathrm{X}$ & & \\
Tioguanina & $\mathrm{X}$ & & & \\
Mitoxantron & & & & \\
Idarrubicina & & & & & \\
\hline
\end{tabular}

Tabla I. Drogas usadas según patología neoplásica. LLA, leucemia linfoblástica aguda; LMA, leucemia mieloblástica aguda; LFM, linfoma; RB, rabdomiosarcoma; TSNC, tumor sistema nervioso central.

Tabla II. Pacientes totales y con patologías bucales por año. aP $<0.05$, Test de Fisher.

\begin{tabular}{lccccc}
\hline Año & Candidiasis & Mucositis & Lesiones herpéticas & Hemorragias & Pacientes con patología bucal \\
\hline 1997 & $21 / 74(28,3 \%)$ & $23 / 74(31 \%)$ & $11 / 74(14,8 \%)$ & $4 / 74(5,4 \%)$ & $35(47,2 \%)$ \\
2007 & $15 / 74(20,2 \%)$ & $8 / 74(10,8 \%)$ & $11 / 74(14,8 \%)$ & $5 / 74(6,7 \%)$ & $24(32,4 \%)$ \\
\hline
\end{tabular}


RODRIGUEZ, M.; MANRIQUEZ, X.; ROJAS, I. G.; FERNANDEZ, E.; BRETAHUER, U. \& SEPÚLVEDA, E. Estudio comparativo: Prevalencia patologías bucales en pacientes pediátricos oncológicos 1997-2007. Int. J. Odontostomat., 4(2):149-156, 2010.

Muchos autores han reportado la prevalencia de patologías orales en pacientes con patología oncológica en tratamiento (Cho et al.; Sonis \& Fey; Fayle \& Curzon, 1991).

Respecto a la mucositis, algunos autores reportan entre un $40 \%$ a $100 \%$ de pacientes que presentan estas lesiones durante el tratamiento, dependiendo de la dosis y droga utilizada (Sonis et al., 2004) mientras otros hablan de un 31 \% (Raber-Durlacher et al., 2000) acercándose más a nuestro resultado que fue de un $31 \%$ de los pacientes bajo tratamiento quimioterapéutico para el año 1997 y un 10,8\% para el 2007.

La reactivación del VHS es muy común en pacientes que han recibido quimioterapia. Redding (1990) reporta una reactivación del virus entre el 50 a $90 \%$ en pacientes bajo quimioterapia y trasplante de medula ósea, mientras que Barret (1986) detectó el virus herpes simple en un $40 \%$ de los pacientes, los cuales padecían leucemia aguda. En nuestro estudio se observó a un $15 \%$ de los pacientes con lesiones herpéticas en ambos años, cifra que está por debajo de los estudios mencionados anteriormente.

En relación a los síndromes hemorragíparos, en estudios anteriormente realizados a pacientes oncológicos infantiles, éstos se presentaron en un $10 \%$ de los pacientes (Sepúlveda et al., 2000), mientras que en nuestro trabajo los observamos en un 5,4 Y 6,7\% de los pacientes en ambos años.

El porcentaje de pacientes que padeció candidiasis oral fue un $28,3 \%$ el año 1997 , y un 20,2 $\%$ el 2007 , siendo la patología más prevalente en este estudio seguido por mucositis, lesiones herpéticas y por último por los síndromes hemorragíparos. Otro estudio realizado en pacientes pediátricos oncológicos que fueron tratados con quimioterapia y trasplante de médula ósea, reportó un mayor número de pacientes con reactivación de VHS (35\%), seguido de mucositis (34\%) y por último candidiasis (15\%) (Dahllöf et al., 1989).

Al comparar los dos años de nuestro estudio, hubo una disminución del número de episodios de candidiasis y mucositis, se mantuvo el número de episodios de lesiones herpéticas y aumentaron levemente los episodios de síndrome hemorragíparo (Fig. 2)

La tendencia a la baja tanto del número de pacientes con mucositis y candidiasis, la disminución de episodios y la disminución significativa del número to- tal de pacientes con patología oral (1997: 47\%; 2007: $32 \%$ ), se podría deber a múltiples causas. Destacarían entre ellas, la mejor calidad de las drogas quimioterapéuticas, el uso de drogas estimuladoras de la médula ósea, mayor control clínico, concientización del resto de los profesionales respecto a las patologías orales y por último la detección precoz y la aplicación de medidas preventivas. Actualmente para favorecer la recuperación de la fórmula hematológica post-quimioterapia, es frecuente el uso de factor estimulante de colonias de granulocitos (G-CSF), droga también usada en nuestro grupo en estudio. Está demostrado científicamente que este factor produce granulocitos competentes en pacientes pediátricos que están bajo tratamiento con algún citotóxico por alguna neoplasia (Czygier et al., 2008). Su uso permite contrarrestar la neutropenia producida por la quimioterapia y así se evitan infecciones secundarias, que algunas veces pueden llegar a ser fatales (Liang, 2003).

Además debemos agregar que ha sido un acuerdo del equipo encargado de este grupo de pacientes en estudio, mantener ciertas medidas generales de atención, las que se podrían resumir de la siguiente manera:

Para prevenir la candidiasis, los pacientes hospitalizados deben ser mantenidos con colutorios de clorhexidina al $0.12 \%$ con una frecuencia de 3 veces al día. Si no pueden realizar colutorios se puede usar la clorhexidina en forma de gel. Además, si el paciente tiene un Recuento Absoluto de Neutrófilos (RAN) $<500$, aunque no tenga candidiasis clínicamente, debe recibir tratamiento antifúngico.

Para prevenir gingivorragias, se debe suspender el cepillado dentario si el paciente tiene $<30.000$ plaquetas.

Para detectar precozmente lesiones orales tales como zonas eritematosas (candidiasis, mucositis), ulceraciones (mucositis, lesiones virales, lesiones bacterianas) y pseudomembranas (candidiasis), se debe realizar una observación clínica detallada de la mucosa bucal, especialmente durante los 5-12 días post- quimioterapia, o en cualquier período neutropénico. Además, si se detecta halitosis, se debe sospechar de úlceras o gingivitis.

Para prevenir lesiones herpéticas, si el paciente presenta una úlcera sospechosa de lesión herpética y tiene un recuento linfocitario $<600$ y fiebre, se debe iniciar terapia con antiviral. 
RODRIGUEZ, M.; MANRIQUEZ, X.; ROJAS, I. G.; FERNANDEZ, E.; BRETAHUER, U. \& SEPÚLVEDA, E. Estudio comparativo: Prevalencia patologías bucales en pacientes pediátricos oncológicos 1997-2007. Int. J. Odontostomat., 4(2):149-156, 2010.

Apenas se detecte cualquier lesión debe ser tratada a la brevedad, teniendo presente el recuento hematológico y el periodo post quimioterapia en que se encuentre.
En conclusión, por la importancia que revisten las patologías orales en el tratamiento de quimioterapia, es recomendable seguir realizando estudios como éste, para disminuirlas, prevenirlas, diagnosticarlas y tratarlas prontamente.

RODRIGUEZ, M.; MANRIQUEZ, X.; ROJAS, I. G.; FERNANDEZ, E.; BRETAHUER, U. \& SEPÚLVEDA, E. Comparative study: oral pathologies prevalence in pediatric oncology patients 1997- 2007. Int. J. Odontostomat., 4(2):149-156, 2010.

ABSTRACT: Pediatric oncology patients frequently have oral lesions due to malignancy or as a side effect of treatment. The aim of this study was to compare the prevalence of oral pathologies in oncology patients hospitalized and treated at the Regional Hospital of Concepción, Chile, in the years 1997 and 2007. A retrospective study was carried out in 74 patients each year. Patients suffered from acute lymphoblastic leukemia, acute myeloblastic leukemia, central nervous system tumors, lymphomas and other neoplasms. General data (age, gender, oncologic disease) and presence of oral pathologies (candidiasis, mucositis post-chemotherapy, herpetic lesions and hemorrhage) were obtained from their clinical records. Data was analyzed for statistical differences. A significant reduction in the number of patients with oral pathologies was found in 2007 in comparison to 1997 ( $P<0.05$, Fisher's test). In addition, candidiasis and oral mucositis showed less prevalence in 2007 as compared to 1997, although no significant differences were found. For the relevance of oral pathologies in the chemotherapy it's important to continue studies about prevention, early detection and treatment of oral pathologies.

KEY WORDS: oral pathologies, pediatric oncology patients, chemotherapy.

\section{REFERENCIAS BIBLIOGRÁFICAS}

Alberth, M.; Majoros, L.; Kovalecz, G.; Borbás, E.; Szegedi, I. J.; Márton, I. \& Kiss, C. Significance of oral candida infections in children with cancer. Pathol. Oncol. Res., 12(4):237-41, 2006.

Barrett, A. P. A long-term prospective clinical study of orofacial herpes simplex virus infection in acute leukemia. Oral Surg. Oral Med. Pathol., 61(2):14952, 1986.

Castell, A. P.; Basté, M. A.; Creus, V. M.; Del Pino, G. B.; Gómez, B. C.; Gómez, G. A.; Gorgas, T. M. Q.; Muro, P. N.; Pellicer, J. M. A.; Sotoca, M. J. M.; Trullàs, A. M. \& Vallés, F. R. Prevención y tratamiento de la mucositis en el paciente oncohematológico. Farmacia Hosp., 25(2):139-49, 2001.

Cerda, J. L.; Romero, M. I. \& Wietstruck, M. A. P. Mortalidad por cáncer infantil en Chile. Modelo de transición epidemiológica en la Infancia. Rev. Chil. Pediatr., 79(5):481-7, 2008.

Cheson, B. D. What Is new in lymphoma?. CA Cancer J. Clin., 54:260-72, 2004.

Cho, S. Y.; Cheng, A. C. \& Cheng, M. C. K. Oral care for children with leukaemia. Hong Kong Med. J., 6:203-8, 2000.
Connor, T. H. \& McDiarmid, M. A. Preventing Occupational Exposures to Antineoplastic Drugs in Health Care Settings. CA Cancer J. Clin., 56:35465, 2006.

Czygier, M.; Dakowicz, L. \& Szmitkowski, M. The effect of granulocyte colony-stimulating factor (G-CSF) on the activity of granulocyte enzymes in children with cancer who developed neutropenia after chemotherapy. Adv. Med. Sci., 53(2):278-82, 2008.

Dahllöf, G.; Heimdahl, A.; Modéer, T.; Twetman, S.; Bolme, P. \& Ringdén, O. Oral mucous membrane lesions in children treated with bone marrow transplantation. Scand. J. Dent. Res., 97(3):26877, 1989.

Del Pozo, H. \& Beresi, V. Quimioterapia en los tumores malignos de la infancia. Rev. Chil. Pediatr., 47(1):1-27, 1976.

Departamento de Epidemiología. Ministerio de Salud, Gobierno de Chile, 2005. Disponible en: epi.minsal.cl/cdvida/htm/Taller2005/0204 carlosbecerra.ppt.

Diamond, H. D. Treatment of Leukemia. CA Cancer J. Clin., 9:54-59, 1959. 
RODRIGUEZ, M.; MANRIQUEZ, X.; ROJAS, I. G.; FERNANDEZ, E.; BRETAHUER, U. \& SEPÚLVEDA, E. Estudio comparativo: Prevalencia patologías bucales en pacientes pediátricos oncológicos 1997-2007. Int. J. Odontostomat., 4(2):149-156, 2010.

Epstein, J. B.; Tsang, A. H. F.; Warkentin, D. \& Ship, J. A. The role of salivary function in modulating chemotherapy-induced oropharyngeal mucositis: A review of the literature. Oral Surg. Oral Med. Oral Pathol. Oral Radiol. Endod., 94:39-44, 2002.

Epstein, J. B.; Hancock, P. J. \& Nantel, S. Oral candidiasis in hematopoietic cell transplantation patients: an outcome-based analysis. Oral Surg. Oral Med. Oral Pathol. Radiol. Endod., 96:154-63, 2003.

Fayle, S. A. \& Curzon, M. E. Oral complications in pediatric oncology patients. Pediatr. Dent., 13(5):289-95, 1991.

Freeman, M. L. M.; Sheridan, B. S.; Bonneau, R. H. \& Hendricks, R. L. Psychological stress compromises CD8_ T cell control of latent herpes simplex virus type 1 infections. J. Immunol., 179:322-8, 2007.

Gozdasoglu, S.; Ertem, M.; Buyukkececi, Z.; Yavuzdemir, S.; Bengisun, S.; Ozenci, H.; Tacyildiz, N.; Unal, E.; Yavuz, G.; Deda, G. \& Aysev, D. Fungal colonization and infection in children with acute leukemia and linfoma during induction therapy. Med. Pediatr. Oncol., 32(5):344-8, 1999.

Herget, G. W.; Riede, U. N.; Schmitt-Gräff, A.; Lübbert, M.; Neumann-Haefelin, D. \& Köhler, G. Generalized herpes simplex virus infection in an immunocompromised patient-report of a case and review of the literature. Pathol. Res. Pract., 201:1239, 2005.

Lalla, R. V.; Sonis, S. T. \& Peterson, D. E. Management of oral mucositis in patients who have cáncer. Dent. Clin. North Am., 52(1):61-77, 2008.

Liang, D. C. The role of colony-stimulating factors and granulocyte transfusion in treatment options for neutropenia in childrens with cancer. Paediatr. Drugs, 5(10):673-84, 2003.

Liu, T.; Khanna, K. M.; Chen, X.; Fink, D. J. \& Hendricks, R. L. CD8_T cells can block herpes simplex virus type 1 (HSV-1) reactivation from latency in sensory neurons. J. Exp. Med., 191:1459-66, 2000.

Miller, C. S. \& Danaher, R. J. Asymptomatic shedding of herpes simplex virus (HSV) in the oral cavity. Oral Surg. Oral Med. Oral Pathol. Oral Radiol. Endod., 105:43-50, 2008.
Ministerio de Salud. Guía clínica: Leucemia en menores de 15 años. Reforma de Salud. Santiago, Gobierno de Chile, 2005.

Ministerio de Salud. Protocolo AUGE: Cáncer Infantil Linfomas y Tumores Sólidos. Santiago, Gobierno de Chile, 2004.

Oeffinger, K. C. \& Hudson, M. M. Long-term Complications Following Childhood and Adolescent Cancer: Foundations for Providing Risk-based Health Care for Survivors. CA Cancer J. Clin., 54:208-36, 2004.

Raber- Durlacher, J. E.; Weijl, N. I.; Abu Saris, M.; De Koning, B.; Zwinderman, A. H. \& Osanto, S. Oral mucositis in patients treated with chemotherapy for solid tumors: a retrospective analysis of $150 \mathrm{ca}$ ses. Support. Care Cancer, 8(5):366-71, 2000.

Redding, S. W. Role of herpes simplex virus reactivation in chemotherapy - induced oral mucositis. $\mathrm{NCl}$ Monogr., 9:103-5, 1990.

Saral, R. Oral complications of cancer therapies. Management of acute viral infections. NCI Monogr., 9:107-10, 1990.

Sepúlveda, E.; Brethauer, U.; Morales, R. \& Jiménez, M. Manifestaciones orales en pacientes pediátricos con patología oncológica. Medicina Oral., 5:193-7, 2000.

Sepúlveda, E.; Rojas, I.; Brethauer, U.; Maulén, N.; Muñoz, M.; Kirsten, L.; Oñate, A.; Fernández, E.; Le Fort, P. \& Rojas J. Effect of white cell counts on the presence of human herpes simplex virus type1 in saliva of pediatric oncology patients. Oral Surg. Oral Med. Oral Pathol. Oral Radiol. Endod., 105:583-8, 2008.

Sonis, S. T.; Elting, L. S.; Keefe, D.; Peterson, D. E.; Shubert, M.; Hauer-Jensen, M.; Nebiyou, B. B.; RaberDurlacher, J.; Donnelly, J. P.; Rubenstein, E. B. \& Mucositis Study Section of the Multinational Association for Supportive Care in Cancer; International Society for Oral Oncology. Perspective on cancer therapy-induced mucosal injury, pathogenesis, measurement, epidemiology, and consequences for patients. Cancer, 100:1995-2025, 2004.

Sonis, S. T. \& Fey, E. G. Oral complications of cáncer therapy. Oncology, 16(5):680-6, 2002. 
RODRIGUEZ, M.; MANRIQUEZ, X.; ROJAS, I. G.; FERNANDEZ, E.; BRETAHUER, U. \& SEPÚLVEDA, E. Estudio comparativo: Prevalencia patologías bucales en pacientes pediátricos oncológicos 1997-2007. Int. J. Odontostomat., 4(2):149-156, 2010.

Subramaniam, P.; Babu, K. L. \& Nagarathna, J. Oral manifestations in acute limphoblastic leukemic children under chemotherapy. J. Clin. Pediatr. Den., 32(4):399-24, 2008.

Ultmann, J. E. The Management of Lymphoma. CA Cancer J. Clin., 21:342-59, 1971.
Dirección para correspondencia:

Ester Sepúlveda T.

Departamento de Patología y Diagnóstico

Facultad de Odontología, Universidad de Concepción

Concepción - CHILE

Email: esepulve@udec.cl

Recibido : 16-12-2009

Aceptado: 22-03-2010 